\title{
IVF with donor eggs over 40 and other health issues impacting IVF success rates
}

\section{What impacts IVF success rates?}

In case of female fertility patients over the age of 40 IVF using donor eggs is highly recommended as it is a treatment that results in high success rates if conducted properly. However, it may happen that apart from infertility the patient suffers from another health disorder which may impact the result of the egg donation treatment. What are they and what fertility doctors and embryologists can do to help these patients achieve healthy pregnancy and live birth?

\section{Hydrosalpinx and IVF results}

IVF and Hydrosalpinx is a medical term for the condition of blocked fallopian tube(s) and fluid in the tubes. It does impact the health status of the IVF patient. Many studies show that blocked fallopian tubes are associated with poor outcome as far as IVF fertilization is concerned. ${ }^{1}$ According to dr Uljana Dorofeyeva, in the case of IVF with donor eggs it recommended to have the Hydrosalpinx removed. The presence of hydrosalpinx can impede fertility in several ways. On a simple biomechanical level, when a tube is blocked by fluid and swelling, the egg cannot travel through and sperm are unable to move normally to meet the egg for fertilization. Even if only one tube is affected, the tubal fluid from that tube may accumulate in the uterus and prevent implantation of embryos or be toxic to the embryo. Generally speaking, medical professionals refrain from performing an embryo transfer before blocked fallopian tubes are removed via laparoscopy.

In a case of hydrosalpinx, the tubes are blocked, and a watery clear liquid begins to collect in the tube, causing it to swell and become distended. Most commonly, the blockage seals off the end of the tube that is closest to the ovary (known as the fimbrial end,) but in some rare cases both ends may be sealed. One or both tubes may be affected. It is a relatively common reason for infertility, and can be caused by many different conditions, including:

a. A previous pelvic infection, such as chlamydia, gonorrhoea, or pelvic inflammatory disease (PID).

b. Endometriosis

c. Adhesions, scarring, or inflammation from previous abdominal surgery

\section{d. Tumors (rarely)}

While IVF bypasses the fallopian tubes entirely and places an embryo right into the uterus, the chances of a successful IVF cycle in women with untreated hydrosalpinx are not encouraging. Patients with untreated hydrosalpinx who undergo IVF have less than half the rate of pregnancy of IVF patients who do not have hydrosalpinx. They also suffer more miscarriages. This may be caused by several factors:

The hydrosalpinx fluid is toxic. This means that even with IVF, the presence of hydrosalpinx may harm the health of the embryo. The fluid may interfere with the lining of the uterus, making it less receptive to an implanting embryo. The trickle of fluid into the uterus may mechanically wash out transferred embryos.
Volume 5 Issue 5 - 2018

\author{
Boris Becker \\ CEO, IVF Media, Germany
}

Correspondence: Boris Becker, CEO, IVF Media, Germany,

Email bores@ivfmedia.org

Received: July 30, 2018 | Published: October 22, 2018

\section{IVF other health issues}

\section{IVF and rheumatoid arthritis}

Rheumatoid arthritis also touches infertility patients and can play a negative role in IVF treatment. Treating such patients requires additional medical investigation. Why? In almost $90 \%$ cases of patients with rheumatoid arthritis doctors find some immune system malfunction or additional immune suppression. That is why before IVF treatment commences immune treatment should be planned and performed for these patients.

\section{IVF and borderline tumour}

Can patients who have lost their ovaries due to tumour but still have their uterus undergo successful IVF treatment using donor eggs? In such patient case the fertility specialist should work with the patient's oncologist in order to plan the treatment accordingly. It is recommended to have IVF 2 years after the cancer free period (remission). The protocol for post-cancer patients undergoing egg donation treatment is not that much different from the protocol of other patients. The hormonal preparation for donor egg embryo transfer in case of patients without ovaries is minimal and requires low dosage hormonal replacement (estrogen) therapy.

\section{IVF and diminished ovarian reserve}

In case of patients with low ovarian reserve IVF treatment using own oocytes can be a lengthy process and it is associated with low success rates. On average, in order to have one blastocyst created we need 6-8 fresh eggs from the patient. According to statistics patients with diminished ovarian reserve are able to provide only 2-3 eggs during a single hormonal stimulation. This means we would require, on average, 3 stimulation cycles in order to achieve the minimal number of eggs (6-8) that would in turn produce one blastocyst. There is no guarantee that this single blastocyst will be euploid either. The alternative treatment for patients with low ovarian reserve is fresh or frozen egg donation program. Fertility doctors recommend this treatment as it offers high success rates and quicker results. With egg donation program patients are able to use 10 or more donor eggs. In order to find a suitable and healthy donor it is recommended to use a reliable egg donation database available online. ${ }^{2}$

\section{Conclusion}

There are many factors, except embryo quality, that may affect 
the success rates of egg donation program. Some of them can be eliminated with the use of surgery (e.g. Hydrosalpinx - laparoscopy), but other factors require more in-depth investigation and, often, additional treatment, like in the case of rheumatoid arthritis (immune system treatment). In case of tumors or cancer a close cooperation between an oncologist and a fertility doctor is required. IVF with donor eggs may often need a more broader approach than just looking at the embryo quality and endometrium lining condition.

\section{Acknowledgements}

I would like to thank dr Uljana Dorofeyeva from Intersono IVF Clinic for her participation in the educational webinar which supported my research and mini review. I am also grateful to the researchers from IVF Media (EggDonationFriends) who work closely with medical professionals to gather and publish reliable information on in vitro fertilization and egg donation.

\section{Conflict of interest}

None.

\section{References}

1. Strandell A, Lindhard A, Waldenström U, et al. Hydrosalpinx and IVF outcome: a prospective, randomized multicentre trial in Scandinavia on salpingectomy prior to IVF. Hum Reprod. 1999;14(11):2762-2769.

2. Dr Uljana Dorofeyeva, Intersono, live webinar. 